\title{
Digital Separation of Occluded Seeds using Image Analysis
}

\author{
Archana Chaugule \\ DYPIET, \\ Pimpri Pune, India
}

\author{
Suresh N. Mali \\ DYPIET, \\ Pimpri Pune, India
}

\begin{abstract}
The shape and size estimation done without separating the touching and overlapping seeds lead to inaccurate values of size and shape. The watershed segmentation and morphological processing suffers from problems like over segmentation and large processing times respectively. An algorithm based on concavities is developed and tested for segmentation of occluded paddy grains. The first step distinguished each seed in a binary image of a grain sample as either an isolated seed or a group of occluded seeds by using the shape properties. The next few steps separated individual seeds in binary images of occluded kernels. Split lines were drawn by the algorithm through the split points which were determined by evaluating the concavity of the corner points detected along the boundary, and selecting those points at which the concavity is highest. This approach is compared with morphological operations and watershed segmentation and the obtained results show that this method is effective in separating the touching seeds. And also after the separation the shape features extracted do not differ a lot from the actual shape features.
\end{abstract}

\section{Keywords}

Concavity, Occluded seeds, Segmentation, Split line and Watershed

\section{INTRODUCTION}

The agronomic traits like seed shape and size affect yield, eating quality and market price therefore are most important [1] from measurement point of view. There is little difference in size among seeds from one plant therefore to obtain accurate seed size a large number of measurements are needed [1]. There is a Correlation between average seed area and average seed weight for various accessions of Arabidopsis [2]. The projected seed area is in most cases very good measurement parameter by itself, related to parameters such as weight or size [3]. The measurement of the area of irregularly shaped raw foods can be tedious [4].

Identification of a grain type from digital images is done by placing the grains in a non-touching style in most of the published researches. To do this there has to be a controlled situation, like a research lab, but is difficult to implement it on site [5].

Images of non-touching corn kernels were acquired for variety identification of five China corn varieties [6]. Kernels were positioned in non-touching fashion, with a mean distance of at least $3 \mathrm{~mm}$ resulting in grain distance of at least $50 \%$ of the average grain's width [7].

While performing image analysis it is often observed that the objects in the image are occluded. Separating individual objects from the occluded seeds using basic morphological operations or general image segmentation methods is not possible as it fails to separate the occluded objects. Due to this reason, some splitting method is needed which will give low number of over and under segmented seeds.

Therefore, the objective of this research was to:

1. Find the performance of watershed segmentation and morphological operations.

2. Develop an algorithm to identify occluded seed from groups of kernels in an image.

3. Develop an algorithm to segment the occluding groups of grain kernels, and

4. Compare the performance of our method with watershed segmentation and morphological operations.

5. Extract the shape features and compare these features with the actual features.

\section{RELATED WORK}

Substantial work in detecting concavity using image processing has been carried out. The theory related to concavity point based methods and the work related to it in detecting concavity is presented.

\subsection{Concavity point based methods}

Concavity points are the boundary points of clustered convex objects which are formed due to touching, merging or overlapping of two or more objects. These are points with high concaveness and high curvature value [8,9]. Figure 1 shows the two occluded seeds and the points of contact at the boundary that are highlighted are the concavity points. Concavity based methods try to impersonate the human approach of separating occluded objects by finding some prominent points on the contour and then finding pairs that satisfy a certain set of conditions and drawing a line between those pairs. There are different methods which vary in how those points are found. 


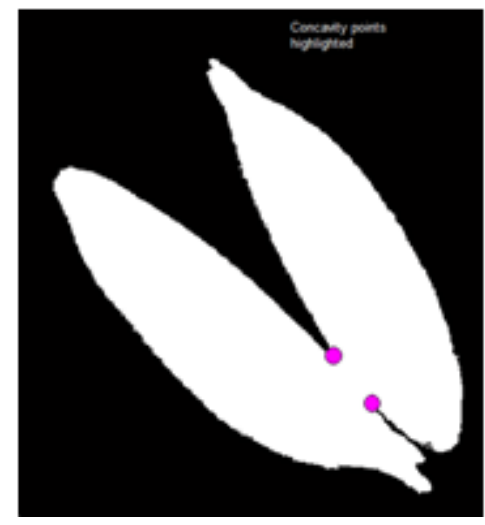

Figure 1 Occluded Seeds and Concavity Points

For the problem of adhesion and occlusion phenomena, Yutan Wang et al. [10] combined the watershed transformation with distance and gradient algorithm to solve over-segmentation and under-segmentation. Yibo Qin et al. [11] proposed a modified watershed segmentation algorithm based on the extended-maxima transform. Juan Feng et al. [12] Proposed Adjacent apples recognition based on chain code information and centroid coordinates. Quan Longzhe et al. [13] made improvement to the watershed algorithm to resolve the problems of algorithm-over-segmentation and leakage, and realized automatic segmentation of touching corn kernels.

Qufa Zhong[14] proposed a novel algorithm based on watershed and concavities to segment the clustered slender particles. The mask is used to determine the concaveness along the whole boundary of touching particles. Arc length and perimeter of circular mask is used to calculate the concaveness. Gao Hua et al. [15] used a chain code difference judgement method in order to search concave point in boundary image. E.H.Van den Berg et al. [16] found the wedge pixel by comparing the dimensionless eccentricity with critical eccentricity.

W. Wang et al. [17] developed a modified morphological watershed segmentation algorithm to overcome the oversegmentation problem that is inherent in the original method. The algorithm was not as capable for separating grain kernels of elongated or complex shapes. N. S. Visen et al. [18] developed algorithm to separate individual grain kernels in binary images of touching kernels. Segmentation lines between nodal points were drawn by the algorithm. Nodal points were determined by evaluating the curvature along the boundary and selecting those points at which the curvature fell below a threshold. It failed in three situations, when the curvature at an actual node was not sharp enough, when pixels on the boundary which were not nodes are misidentified as nodes because of roughness, and when the nearest-neighbour criterion did not yield the correct segmentation line.

To find the line between concavity points for segregation of clumped objects in an image G. Feranadez [8] proposed the clump splitting algorithm which uses spatial and gradient. The decision between the two concavity points is based on two conditions: the distance between the two concavity points as compared to the perimeter of the clumped object and the flatness of the path between the concavity points. Another method based on searching for convex hull is used for detecting concavity $[19,20]$.

\section{METHOD}

\subsection{Image capturing}

The images were acquired using digital camera from different distances. Randomly any numbers of seeds were placed on the black background with the kernels touching each other.

\subsection{Approaches for separating the occluded seeds}

The approaches that are applied for separating the occluded seeds are

$$
\begin{aligned}
\text { i. } & \text { Morphological operations approach } \\
\text { ii. } & \text { Watershed Segmentation approach } \\
\text { iii. } & \text { Proposed approach }
\end{aligned}
$$

\subsubsection{Morphological operation approach}

Dilation is an operation that thickens objects in a binary image. On the other hand erosion shrinks the objects. This thickening and thinning is controlled by the structuring element. The erosion operation is useful for removing small objects. However, it has the disadvantage that all the remaining objects shrink in size. We can avoid this effect by dilating the image after erosion with the same structure element. This combination of operations is called an opening operation

The morphological opening of $\mathrm{A}$ by $\mathrm{B}$ denoted by $\mathrm{A}{ }^{\circ} \mathrm{B}$ is simply erosion of $\mathrm{A}$ by $\mathrm{B}$, followed by dilation of the result by $\mathrm{B}$ as shown in (1).

$A^{\circ} B=(A \ominus B) \oplus \quad B$

To separate out the seeds the method we have applied is erosion followed by dilation of the eroded image and then subtraction of eroded from dilated image. The result is as shown in Figure 2. 


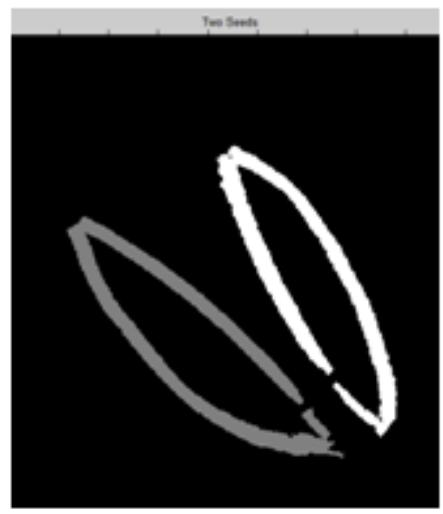

Figure 2 Result of Morphological operations

The Morphological operation approach separates the seeds but shrinks the size and the shape of the seed changes. And the processing time required is relatively large. The other approach tried was watershed segmentation.

\subsubsection{Watershed segmentation approach}

Watershed segmentation is a technique in which continuous boundaries, known as watershed lines are found between the objects that may or may not be touching each other. Watershed segmentation is also a commonly used method for not only segmenting objects from the background but also partitioning touching or overlapping objects from each other [21].

Figure 3 shows the occluded seeds after applying watershed segmentation using the distance transform. The result shows ridges on the seed interior due to over-segmentation. This over-segmentation is due to the existence of false minima in the images.

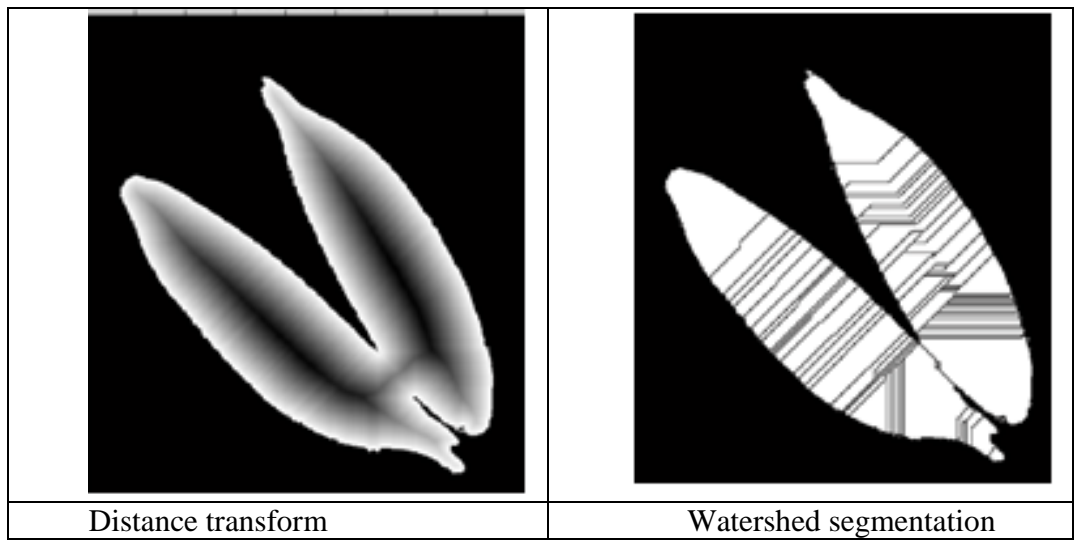

Figure 3 Watershed using the distance transforms

To overcome this over-segmentation problem two techniques as described in [22] are applied. These two techniques are watershed segmentation using Gradients and markercontrolled watershed segmentation.

\subsubsection{Watershed using gradient}

The gradient image has high pixel values along object edges and low pixel values elsewhere. Thus the watershed transform results in watershed ridge lines along object edges. It can be difficult to determine which catchment basins are actually associated with the objects of interest. Direct application of the watershed transform to a gradient image usually leads to over-segmentation due to noise and other local irregularities of the gradient [22]. The result is as shown in Figure 4. Next approach to control over-segmentation is based on the concept of markers.

\subsubsection{Marker modified}

A marker is a connected component belonging to an image. These markers are then used to modify the gradient image [22]. The result is as shown in Figure 4. 


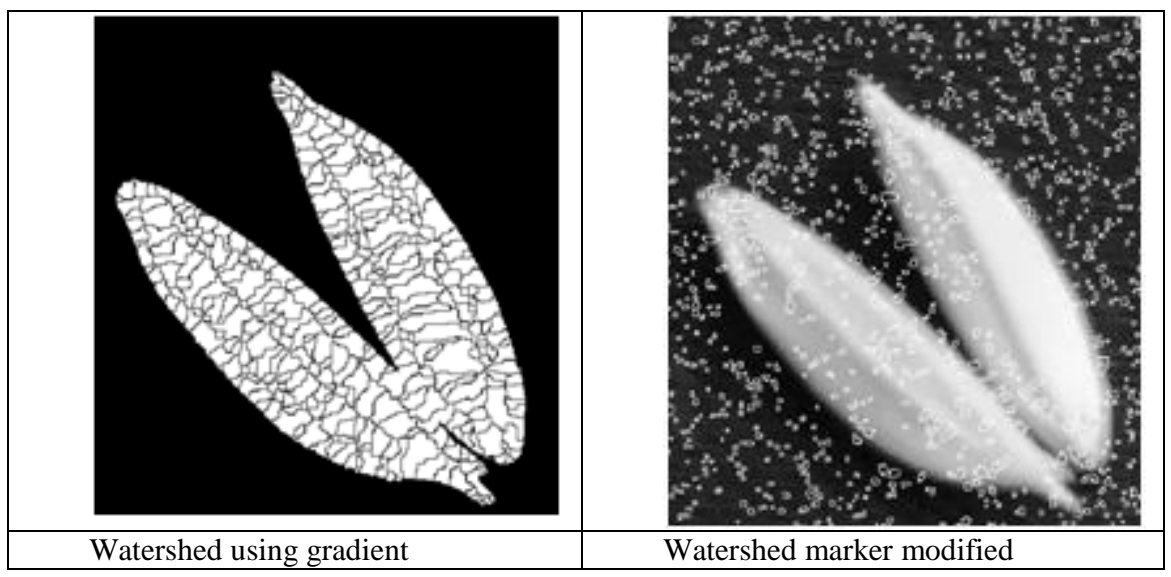

Figure 4 Watershed using Gradient and Marker

From watershed approach it can be seen and also be concluded that the occluded seeds cannot be separated out efficiently.

\subsection{Proposed methodology}

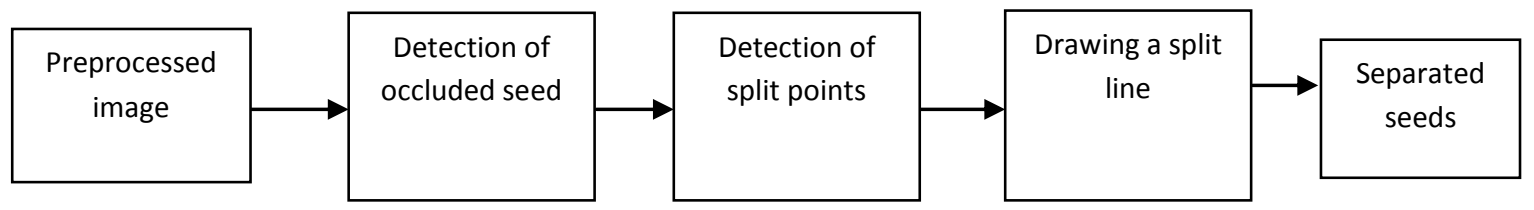

Figure 5 Block Diagram

The block diagram for the proposed methodology is as shown in Figure 5. The steps are as follows:

This method is usually applied to the binary segmented images as a post-processing step. The steps are as follows

1. Detection of occluded seeds

2. Detection of split points

3. Drawing a split line

\subsubsection{Detection of occluded seed}

The first step is to distinguish each seed in a binary image of a grain sample as either an isolated seed or a group of occluding seeds by using the shape properties. The shape property solidity is used to find whether the seed is isolated or occluded. Grains are convex or nearby convex in shape as shown in Figure6.1, but the grain in Figure 6.2 has concave shape as it is occluded.

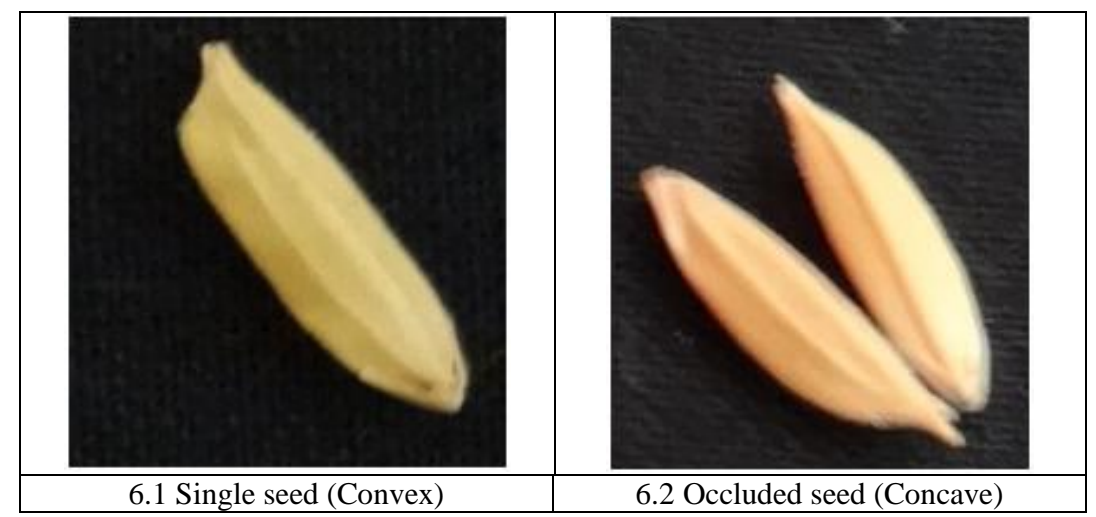

Figure 6 Convex and Concave shape seeds

Solidity describes the extent to which the shape is convex or concave [23]. The solidity of a convex shape is always one. The solidity is given by following formula (2)

solidity $=A_{S} \div H$

Where $A_{S}$ : the area of the shape region
$H$ : the convex hull area of the shape.

The solidity of seed shown in Figure 6.1 calculated by (4) is nearly 1 but of the seed shown in Figure 6.2 is equal to 0.7799 . Thus solidity is used to find whether the seed is concave or convex in shape. 


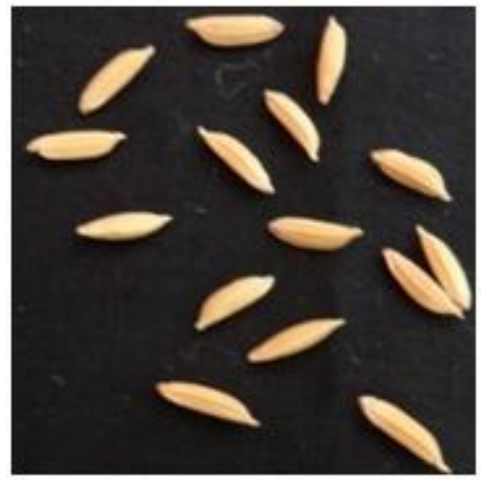

Figure 7 Group of Seeds

So from a group of seeds as shown in Figure 7, occluded seeds can be found out by using solidity as follows

If solidity $(\mathrm{K})<0.90$ then

$$
\mathrm{K}^{\text {th }} \text { seed is occluded (concave) }
$$

Else

$$
\mathrm{K}^{\text {th }} \text { seed is not occluded (convex) }
$$

endif

\subsubsection{Detection of split points}

Once the occluded seed is found from the group of seeds the next step is to find the split points to separate individual seeds in binary images of occluded kernels. The split point is found by first detecting the corner points on the boundary of seed. Before detecting the corner points the image is first rotated so that it is orientated vertically. In this algorithm, in order to search the corner point along continuous boundary, the image boundary is found and an approach based on global and local curvature properties has been used to detect corners as described in [24]. Algorithm to find corner points is as follows [24]:

i. Apply the Canny edge detector to the gray level image and obtain a binary edge-map.

ii. Extract the edge contours from the edge-map, fill the gaps in the contours, if the endpoint of a contour is nearly connected to another endpoint, fill the gap and continue the extraction. The default gap size is 1 pixles.

iii. Compute curvature at a low scale for each contour to retain all true corners. iv. All of the curvature local maxima are considered as corner candidates, then rounded corners and false corners due to boundary noise and details were eliminated.

v. End points of line mode curve were added as corner, if they are not close to the above detected corners.

Once the corner points are detected a mask operator is used to determine the significance of a concavity. To reduce the processing time only the detected corners are used instead of the whole contour for determining concavity.

To detect the concave point a method based on burning the image with the help of a circular mask is developed. The centre of circular mask with a radius of 30 pixels is placed on the corners of the seed as shown in Figure 8. Only the part of the image that is inside the mask is kept and the part outside the mask is removed.

$$
\text { burnedImage }(\text { not binaryimage })=255
$$

The part of the image that is kept is called burned-image and the area of the burned-image is used to calculate the concavity of every corner point. Thus Concavity is expressed as

Where,

$$
\begin{gathered}
\text { concavity_cornerpoint }(i)=\text { area_burnedimage }(i) \div \\
\text { perimeter_mask }
\end{gathered}
$$

area_burnedimage(i) is the area of the burned image of $i^{\text {th }}$ corner point perimeter_mask is the perimeter of the circular mask

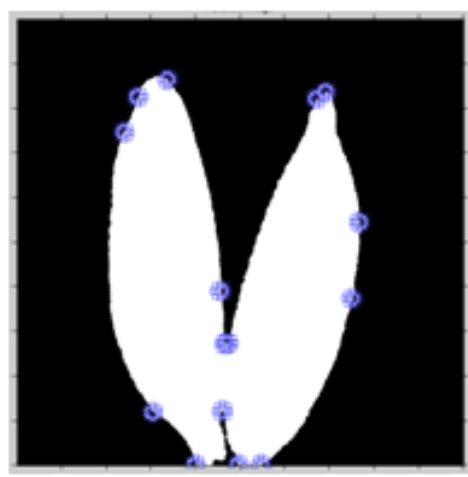

Figure 8 Detection of Split Points 


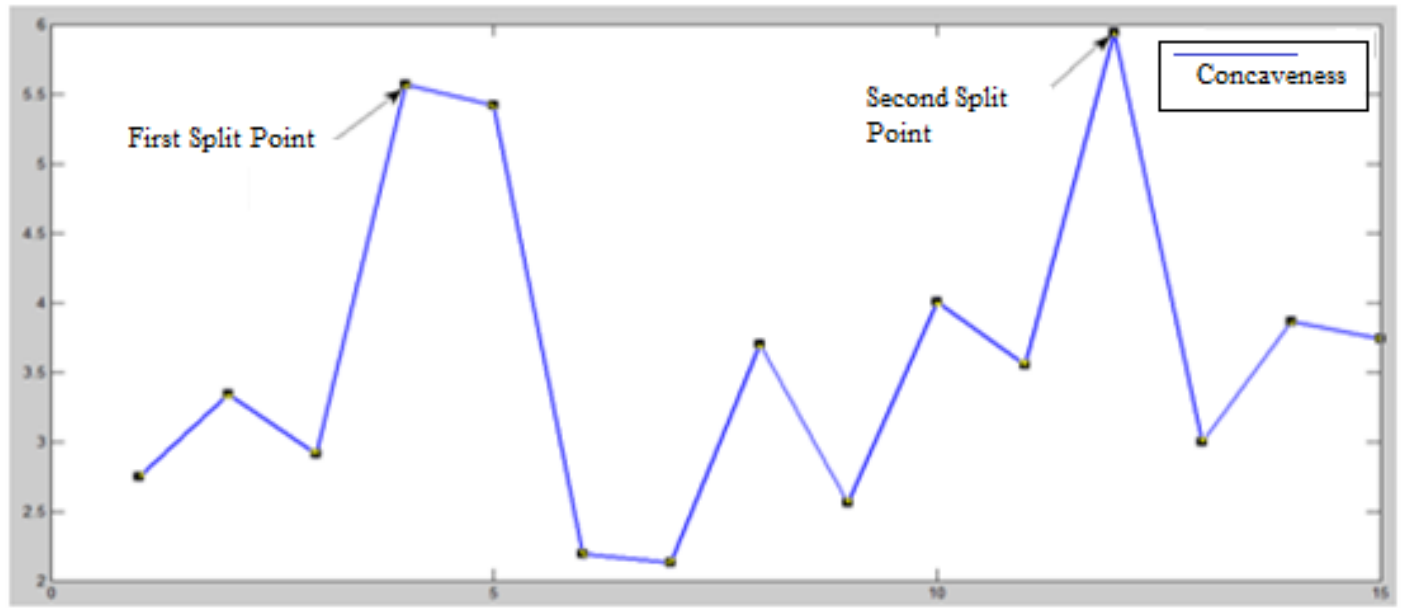

Figure 9 Plot of Concavity

The concavity of the split point has maximum value as shown in Figure 9, so first two points having maximum concavity are taken and they are treated as split points.

\subsubsection{Drawing a split line}

These split points are then joined by a split line thus splitting the two occluded seeds as shown in Figure 10. The split line is drawn as follows: the $\mathrm{x}$ and $\mathrm{y}$ coordinates of the two points are taken and according to equation of line $y=m \times x+c$

(5)

The line is drawn between these two points.

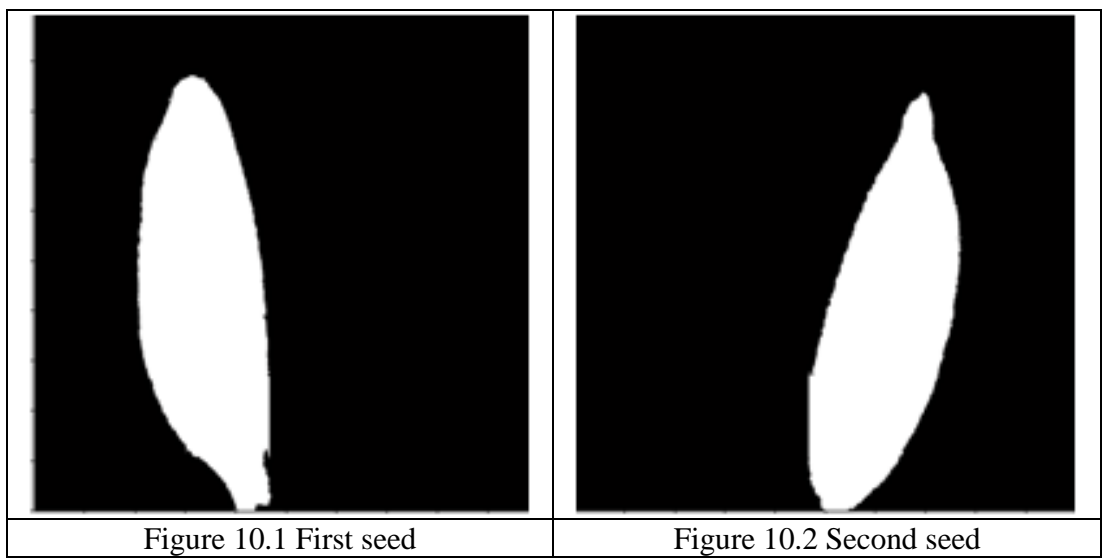

Figure 10 Separations of Occluded Seed

\section{RESULTS AND DISCUSSIONS}

The Set-1 used is as shown in Figure 7. The total number of seeds used are fifteen, but as there is one occluded pair, the total number of seeds considered during calculation are fourteen. So the calculation of shape features differ, as it considers the occluded seeds as a single seed. The occluded seeds from Figure 7 are shown in Figure 8. By using the proposed method, the occluded seeds are separated and the separation is as shown in Figure 10. Even after separation by the proposed method the shape features calculated do not differ a lot. The comparison of actual features and features calculated by proposed method is as shown in the Table -1 .

Table 1. Comparison of Actual values and proposed method

\begin{tabular}{|l|l|l|l|}
\hline \multirow{2}{*}{ Shape Feature } & \multicolumn{3}{|c|}{ Set-1 } \\
\cline { 2 - 4 } & Actual & Proposed Method & Accuracy \% \\
\hline Area & 32914 & 32873.13333 & 99.88 \\
\hline Perimeter & 981.8793 & 1017.998518 & 96.32 \\
\hline Major-axis & 389.8123 & 381.3471621 & 97.82 \\
\hline Minor-axis & 109.083 & 112.6927081 & 96.70 \\
\hline
\end{tabular}


Table 2. Comparison of Actual values and proposed method and Accuracy of Separated seeds

\begin{tabular}{|l|l|l|l|c|l|l|}
\hline \multirow{2}{*}{ Shape Feature } & \multicolumn{3}{|c|}{ First-Seed } & \multicolumn{3}{c|}{ Second-Seed } \\
\cline { 2 - 7 } & Actual & Proposed Method & Accuracy \% & Actual & Proposed Method & Accuracy \% \\
\hline Area & 36558 & 36287 & 99.26 & 31149 & 32811 & 94.66 \\
\hline Perimeter & 1030.438 & 1102.749 & 93.00 & 1028.48 & 1027.377 & 99.90 \\
\hline Major-axis & 398.8544 & 409.2159 & 97.40 & 393.22 & 389.6862 & 99.10 \\
\hline Minor-axis & 118.6454 & 113.4677 & 95.63 & 104.05 & 108.8127 & 95.42 \\
\hline
\end{tabular}

From Set-1, the seeds separated and as shown in Figure 10, have features calculated as shown in Table-2. The actual values are the values of that seed calculated without touching each other and the proposed method values are the values of the seed after separating the occluded seeds. The accuracy shown for First-Seed is around 93.00 to $99.26 \%$ and for Second-Seed is around 94.66 to $99.90 \%$ for different features.

\section{CONCLUSION AND FUTURE SCOPE}

The overlapping seed separation method is proposed based on split point detection using concavity. The same is compared with morphological operations and watershed segmentation. The results obtained show that the proposed method is effective for separating the occluded seeds than the other two by observing the result in the images. The Comparison of the extracted features for different sets shown in Table-1, gives the accuracy around 96.32 to 99.88 for Set-1. Similarly the Comparison of the extracted features for Separated seeds shown in Table-2, gives the accuracy around 93.00 to $99.26 \%$ for First-Seed and for Second-Seed is around 94.66 to 99.90 $\%$. However the methodology may be extended to find the exact shape of the seed if two seeds are totally or partially overlapped.

\section{REFERENCES}

[1] Takanari Tanabata, Taeko Shibaya, Kiyosumi Hori, Kaworu Ebana, and Masahiro Yano. 2012. SmartGrain: High-Throughput Phenotyping Software for Measuring Seed Shape through Image Analysis, American Society of Plant Biologists, Plant Physiology, 160:1871-1880

[2] Rowan P Herridge, Robert C Day, Samantha Baldwin, Richard C Macknight. 2011. Rapid analysis of seed size in Arabidopsis for mutant and QTL discovery, Plant Methods, 7:3

[3] Scanalyzer Phenotyping. 2008. Seed Phenotyping by Size, Shape And ColourLemnaTec GmbH.

[4] Chaugule A, Mali S. N. 2013. Area Measurement of Seed from Distorted Images for Quality Seed Selection. Nirma University International Conference on Engineering, NUiCONE-2013, 978-1-4799-07274/13/\$31.00 @2013 IEEE , 1-7.

[5] Choudhary R, Paliwal J, Jayas, DS. 2008. Classification of Cereal Grains using Wavelet, Morphological, Colour, and Textural Features Of Non-Touching Kernel Images. Elsevier, Biosystems Engineering 99:330-337.

[6] Chen X, Xun Y, Li W, and Zhang J. 2010. Combining Discriminant Analysis and Neural Networks for Corn Variety Identification. Elsevier, Computers and Electronics in Agriculture 71S:S48-S53.
[7] Piotr M, Szczypin ski, Zapotoczny P. 2012. Computer Vision Algorithm for Barley Kernel Identification, Orientation Estimation and Surface Structure Assessment. Elsevier, Computers and Electronics in Agriculture 87:32-38.

[8] Fernandez G, Kunt M and Zryd JP. 1995. A New Plant Cell Segmentation Algorithm. Proceedings of $8^{\text {th }}$ International conference of image analysis and processing 229-234.

[9] Kumar S, Ong SH, Ranganath S., Ong TC, and Chew FT. 2006. A Rule-Based Approach for Robust Clump Splitting. Pattern Recognition 39(6):1088-1098.

[10] Yutan W, Kan J, Li W and Zhan C. 2013. Image Segmentation and Maturity Recognition Algorithm based on Color Features of Lingwu Long Jujube. Advance Journal of Food Science and Technology ISSN: 20424868; e-ISSN: 2042-4876 5(12): 1625-1631.

[11] Yibo Q, Wang W, Liu W, and Yuan N. 2013. ExtendedMaxima Transform Watershed Segmentation Algorithm for Touching Corn Kernels. Hindawi Publishing Corporation Advances in Mechanical Engineering, $\begin{array}{llll}\text { Article ID } & \text { 268046, }\end{array}$ http://dx.doi.org/10.1155/2013/268046

[12] Feng J, Wang S, Liu G and Zeng L. 2012. A separating Method of Adjacent Apples Based on Machine Vision and Chain Code information. Computer and computing technologies in Agriculture V, IFIP Advances in Information ND Communication technology 368:258267.

[13] Quan L and Jiang E. 2011. Automatic Segmentation Method of Touching Corn Kernels in Digital Image Based on Improved Watershed Algorithm. 978-1-42449577-1/11 34-37.

[14] Zhong Q, Zhou P, Yao Q, Mao K. 2009. A Novel segmentation algorithm for clustered slender particles. Elsevier, Computers and Electronics in Agriculture 69:118-127.

[15] Hua G, Yaqin W, Pingju G. 2007. Research on Segmentation Algorithm of Adhesive Plant Grain Image. The Eighth International Conference on Electronic Measurement and Instruments, ICEMI'2007, 2_9272_930

[16] Van EH , Berg, Meesters AGCA, Kenter JAM., Schlager. 2002. Automated Separation of Touching Grains in Digital Images of Thin Sections. Pergamon, Computers \& Geosciences 28:179-190. 
[17] Wang W and Paliwal J. 2006. Separation and Identification of Touching Kernels and Dockage Components In Digital Images. Canadian Biosystems Engineering 48:7.1-7.7

[18] Visen NS, Shashidhar NS, Paliwal J, Jayas DS. 2001. Identification and Segmentation of Occluding Groups of Grain Kernels in a Grain Sample Image. J. agric. Engng Res. AE*Automation and Emerging Technologies, 79(2):159-166, doi:10.1006/jaer.2000.0690

[19] JinYXC, Jayasooriah SHO,Sinniah R. 1994. Clump Splitting Through Concavity Analysis. Pattern Recognition Letters 15:1013-1018

[20] Rosenfeld A. 1985. Measuring the Sizes of Concavities. Pattern Recognition Letters 3:71-75
[21] Vincent L and soille P. 1991. Watersheds in digital spaces: an efficient algorithm based on immersion simulations. IEEE Transactions on Pattern Analysis and Machine Intelligence 13(6):583-598

[22] Gonzalez RC, Woods RE, Eddins SL. 2010. Digital Image Processing Using Matlab. Tata McGraw Hill Education Private Limited

[23] Mingqiang Y, Kidiyo K and Joseph R. 2008. A Survey of Shape Feature Extraction Techniques. Pattern Recognition Techniques, Technology and Applications, ISBN 978-953-7619-24-4: 626

[24] He XC and Yung NHC. 2008. Corner Detector Based on Global and Local Curvature Properties. Optical Engineering 47(5), 057008_1-057008_12 